\title{
Una nueva especie del género Trapania (Nudibranchia: Goniodorididae) para el Mar Argentino
}

\author{
A new species of the genus Trapania (Nudibranchia: \\ Goniodorididae) from the Argentine Sea
}

\author{
Nicolás Cetra ${ }^{1 *}$, Andrea Roche ${ }^{1,2}$ \\ 1 Escuela Superior de Ciencias Marinas (ESCiMar), San Antonio Oeste, Río Negro, Argentina. \\ 2 Centro de Investigación y Transferencia Tecnológica en Recursos Marinos Almirante Storni (CIMAS), \\ San Antonio Oeste, Río Negro, Argentina. \\ *nicolascetra@gmail.com
}

\section{RESUMEN}

Se describe una especie de gasterópodo marino para el género Trapania, Pruvot-Fol, 1931, a partir de ejemplares colectados en Las Grutas, Río Negro, en el Mar Argentino. Trapania rocheae sp. nov. tiene el cuerpo, los rinóforos y las branquias de color blanco, cubiertos por manchas de color pardo. Presenta un par de procesos extrabranquiales y extrarinofóricos alargados de color blanco los cuales poseen manchas de color pardo. La rádula se compone de un solo diente lateral con pequeñas cúspides que aumentan de tamaño hacia el margen posterior. Este es el primer registro de este género para la Argentina.

Palabras clave - Trapania rocheae sp. nov., Nudibranchia, Mar Argentino.

\begin{abstract}
A new marine gastropod species of the genus Trapania, Pruvot-Fol, 1931, is described from specimens collected from Las Grutas, Río Negro, in the Argentine Sea. Trapania rocheae sp. nov. has a body, rhinophores and gills white color, with some red-brown spots. There is a pair of elongated extratransbranchial and extrarhinophoric white

\footnotetext{
- Ref. bibliográfica: Cetra, N.; Roche, A. 2019. "Una nueva especie del género Trapania (Nudibranchia: Goniodorididae) para el Mar Argentino". Acta zoológica lilloana 63 (2): 1-10. Fundación Miguel Lillo, Tucumán, Argentina. D.O.I.: https://doi.org/10.30550/j.azl/2019.63.2/1

> Recibido: 01/04/19 - Aceptado: 30/09/19

> URL de la revista: http://actazoologica.lillo.org.ar

> Algunos derechos reservados. Esta obra está bajo una Licencia Creative Commons Atribución - No Comercial - Sin Obra Derivada 4.0 Interna-

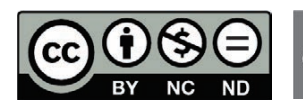


processes with brown spots. The radula is composed of a single lateral tooth with small cusps that increase in size towards the posterior margin. This is the first record of this genus for Argentina.

Keywords - Trapania rocheae sp. nov., Nudibranchia, Argentine Sea.

\section{INTRODUCCIÓN}

Los nudibranquios son moluscos heterobranquios comúnmente conocidos como babosas marinas. Se caracterizan por presentar una concha muy reducida, interiorizada o, como ocurre en la mayoría de los casos, carecer completamente de ella en estado adulto. Exhiben un amplio arreglo de formas corporales, por lo que su anatomía externa puede tener diversos aspectos (Ardila, et al., 2007). Habitan los mares de todo el mundo, desde las aguas tropicales hasta los polos y desde zonas intermareales hasta grandes profundidades (Chavanich, et al., 2013). Entre los nudibranquios, el género Trapania Pruvot- Fol, 1931, se caracteriza por un cuerpo liso, elongado, con dos pares de procesos laterales curvos, uno junto a los rinóforos y otro a las branquias. Los rinóforos son foliados y poseen tres branquias tripinnadas dorsoposteriores. La parte anterior de la cabeza porta un par de tentáculos orales cortos. La fórmula radular es 0.1.0.1.0 y los dientes presentan cúspides que incrementan su tamaño hacia el borde externo. La armadura masticatoria presenta un borde con dentículos irregulares. Este género se encuentra ampliamente distribuido en el mundo (Rudman, 1987; Fahey, 2004), la mayoría de las especies se han documentado en el Atlántico Oriental (Haefelfinger, 1960; Kress, 1968; Cervera y García-Gómez, 1989; Cervera, et al., 2000) y en las regiones templado-tropicales del Indo-Pacífico y Australasia (Miller, 1981; Rudman, 1987; Fahey, 2004). También se han descrito tres especies adicionales del Pacífico Oriental (Camacho-García y Ortea, 2000; Millen y Bertsch, 2000; Hermosillo y Valdés, 2004) y para el Pacífico Occidental (Gosliner y Fahey 2008). En Sudamérica, el género Trapania se encuentra representado por dos especies, Trapania darwini Gosliner y Fahey, 2008 citada en las Islas Galápagos, Ecuador (Gosliner y Fahey, 2008) y Trapania maringa Marcus, 1957 en Ubatuba, Brasil (Marcus, 1957). Particularmente en la Argentina no se conocen registros de especies para este género (Schröldl, 2003; García y Bertsch, 2009).

$\mathrm{El}$ objetivo de este trabajo es dar a conocer una nueva especie, Trapania rocheae sp. nov. colectada en el parque submarino Las Grutas, Río Negro Argentina.

\section{MATERIALES Y MÉTODOS}

\section{Metodología}

Los ejemplares analizados en este estudio fueron colectados de un sitio submareal del Balneario Las Grutas (40 49.291' S; 065 04.498' O) ubicado en el sector noroeste del Golfo San Matías (Figura 1). Los especímenes fueron colectados de forma 


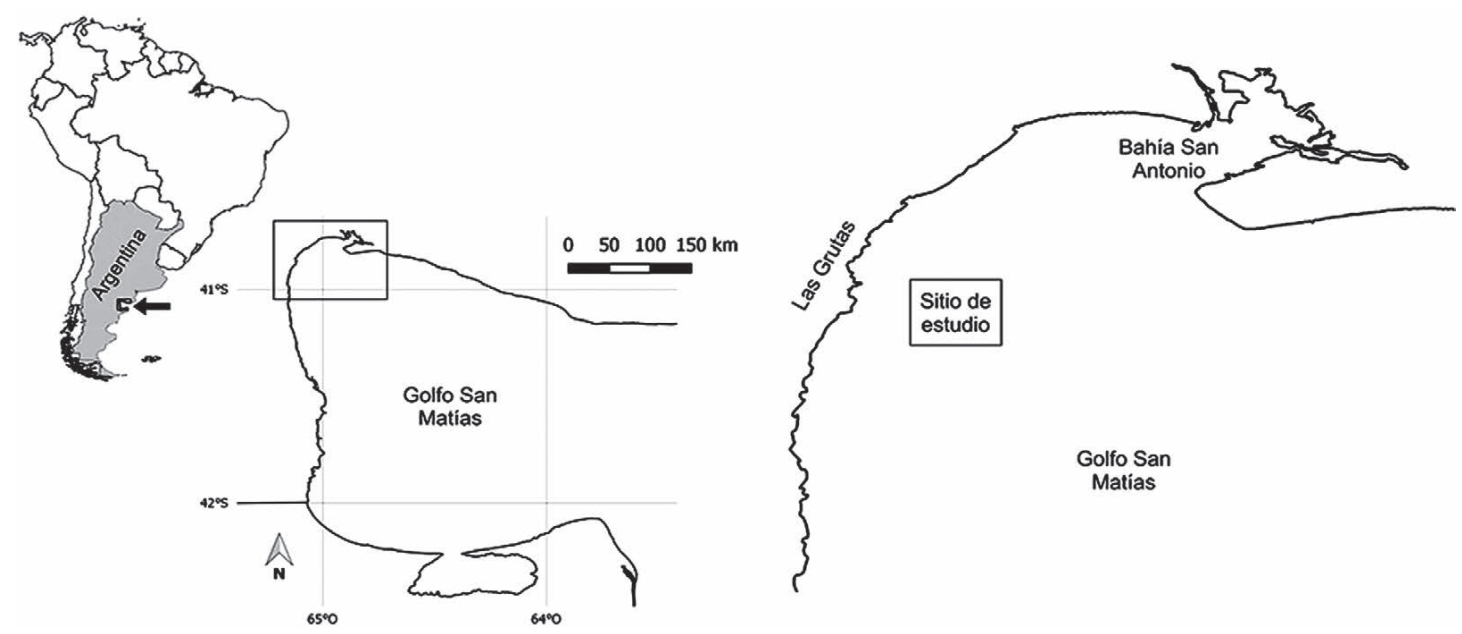

Figura 1. Localización del sitio de estudio, Las Grutas, Golfo San Matías, Argentina.

manual mediante buceo autónomo entre los 4 y 10 metros de profundidad. Durante los buceos se registraron todos los individuos de la especie. A cada espécimen se le tomaron fotografías in situ para registrar el patrón de coloración natural. Con el fin de realizar análisis morfológicos, algunos ejemplares fueron llevados al laboratorio y examinados bajo microscopio estereoscópico, medidos y fotografiados antes de ser conservados en alcohol al 70\%. La rádula y la mandíbula del holotipo fueron separadas de la masa bucal utilizando el método de Holznagel (1998). Ambas fueron montadas y posteriormente fotografiadas utilizando el microscopio de barrido electrónico (JEOL/JSMT 6360 LV, Tokyo, Japan) del Museo de La Plata.

\section{RESULTADOS}

Orden Nudibranchia Cuvier, 1817

Familia Goniodorididae H. Adams y A. Adams, 1854

Género Trapania Pruvot-Fol, 1931

Trapania rocheae Cetra y Roche sp. nov.

(figuras $2,3,4,5$ )

lsid: zoobank.org: pub: 84145485-F43C-4C19-A0AB-5330C141B723

Material tipo.- Holotipo: MLP-Ma 14834, un ejemplar de $9 \mathrm{~mm}$, Balneario Las Grutas, Río Negro, Argentina (40 49.291' S; 065 04.498’ O), 04/04/17. Colectado por buceo autónomo a $8 \mathrm{~m}$ de profundidad sobre roca asociado a hidrozoos.

Localidad tipo.- - Balneario Las Grutas, Río Negro, Argentina (40 $49.291^{\prime}$ S; $065^{\circ}$ 04.498' O).

Diagnosis. - Cuerpo alargado y liso, de coloración blanco pálido con manchas color marrón oscuro que se extienden por todo el cuerpo, incluyendo rinóforos, branquias y procesos extrabranquiales y extrarinofóricos (Figura 2). 


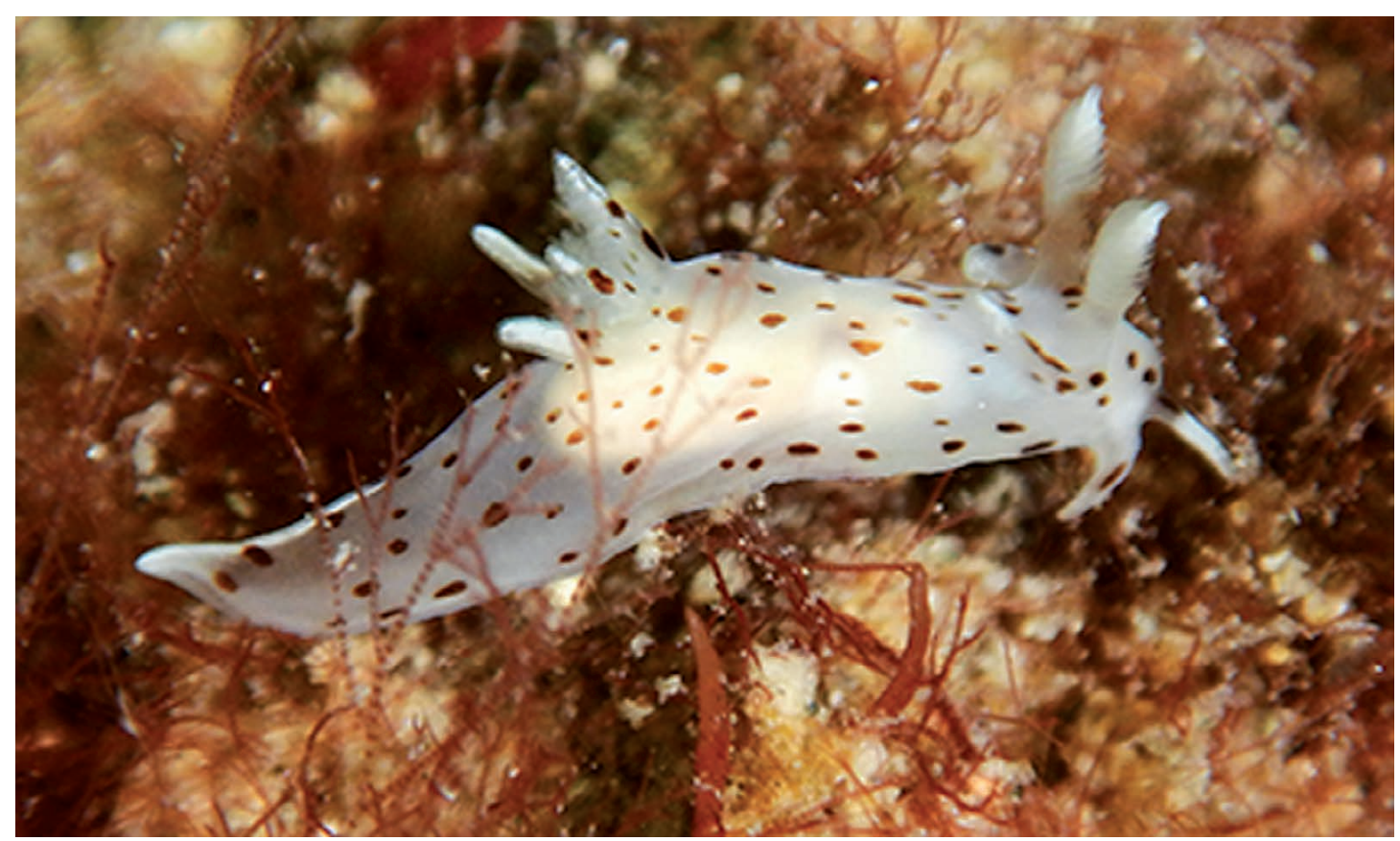

Figura 2. Trapania rocheae sp. nov. en ambiente natural. (Isid: zoobank.org: pub: 84145485-F43C4C19-A0AB-5330C141B723).

\section{Descripción}

Morfología externa.- Los especímenes examinados presentaron pequeño tamaño, entre 8 y $12 \mathrm{~mm}$ de longitud en especímenes vivos (Holotipo: $9 \mathrm{~mm}$ ). Cuerpo alargado y liso, de coloración blanco pálido con manchas color pardo que se extienden por todo el cuerpo, incluyendo rinóforos, branquias y procesos extrarinofóricos y extrabranquiales. Una cresta notal color blanca recorre la zona posterior del dorso desde la base de las branquias hasta el extremo posterior del cuerpo. Presenta dos pares de procesos dorsales, uno frente a los rinóforos (extrarinofóricos) y otro delante de las branquias (extrabranquiales). Tentáculos orales cortos y cónicos en la zona anterior de la cabeza, con manchas pardo-oscuras en la base. Rinóforos foliados hasta 13 veces. Branquias tripinnadas dorso-posteriores, dispuestas en semicírculo alrededor del ano (Figura 3, A). El pie es ancho y redondeado en el área anterior, terminado con una cola en forma de punta en su parte posterior.

Sistema digestivo.- Este sistema es el típico de otras especies del género (Rudman, 1987), el esófago es corto y desemboca en la glándula digestiva; intestino simple y curvo. Bulbo bucal ovalado, rodeado de cuatro grandes músculos. Dos glándulas salivales se ubican en su parte superior, una a cada lado del esófago. Fórmula radular 32 x 0.1.0.1.0 (Figura 3, B). Los dientes laterales son rectangulares, con 8 dentículos grandes y entre éstos, 3 a 6 dentículos más pequeños, última cúspide triangular y alargada en forma de hoz (Figura 3, C). Armadura masticatoria compuesta por cuatro placas rectangulares alargadas, unidas en forma compacta. Borde masticatorio ligeramente curvado con dentículos que disminuyen de tamaño hacia el interior de las placas (Figura 3, D). 

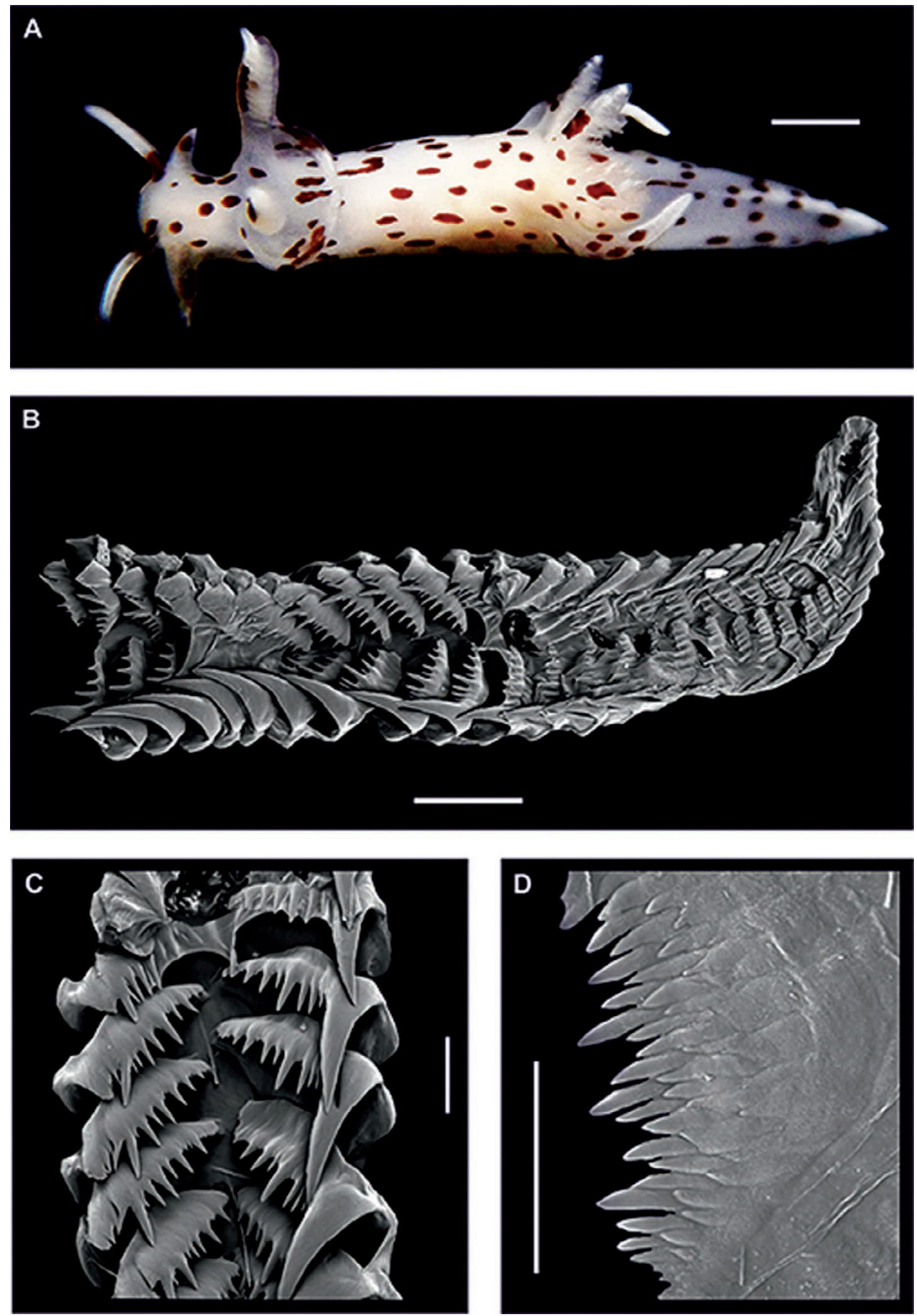

Figura 3. Trapania rocheae sp. nov. A) Espécimen vivo. B) Vista general de la rádula. C) Detalle de los dientes laterales. D) Borde masticatorio de la armadura del disco labial. Barras de escala: A) $1 \mathrm{~mm}$; B y D) $50 \mu \mathrm{m}$; C) $20 \mu \mathrm{m}$. (Isid: zoobank.org: pub: 84145485-F43C-4C19-A0AB-5330C141B723) 
Sistema reproductor.- (Figura 4) Diaulico. Abertura vaginal por debajo de la peneal. Conducto deferente alargado, conectado con la porción distal de la próstata, la cual se ensancha hacia su porción proximal. Próstata junto con la ampulla conectada con la glándula femenina a través de un delicado conducto alargado. Vagina amplia en su abertura, extendiéndose ligeramente formando el conducto vaginal, se une con la bursa copulatriz de apariencia lisa y ovalada. En la base de la bursa copulatriz el conducto vaginal se conecta con el receptáculo vaginal. La porción basal del receptáculo seminal se encuentra conectada con la glándula femenina.

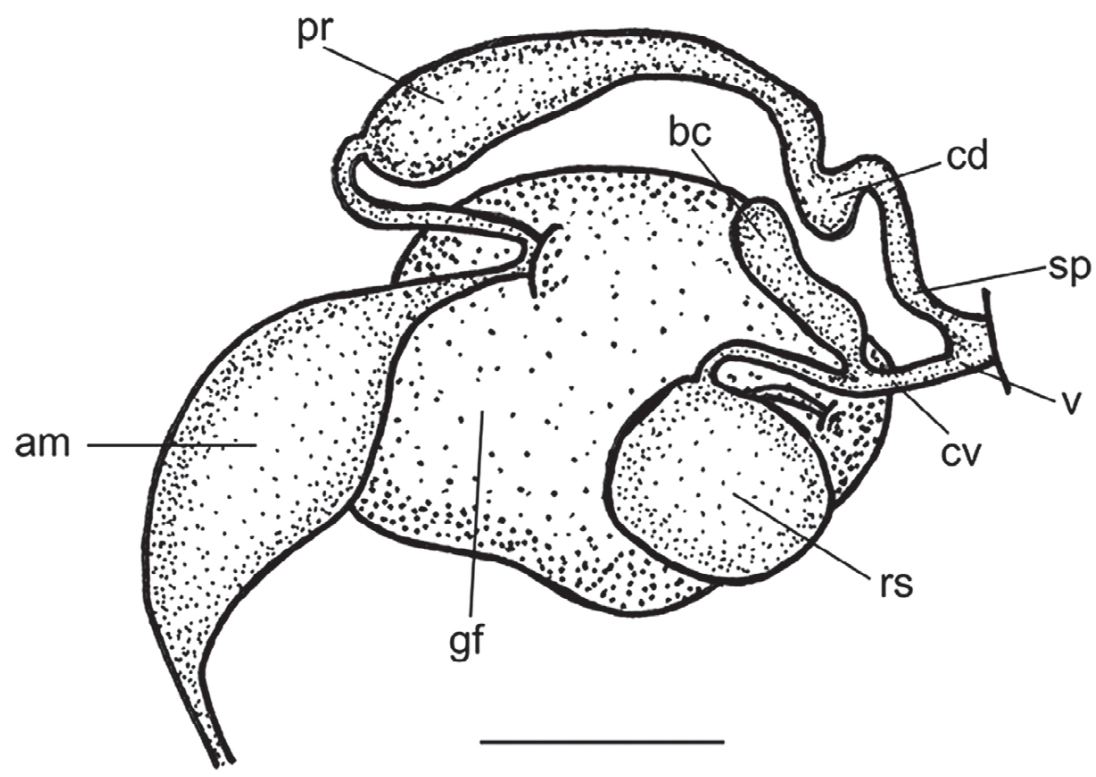

Figura 4. Trapania rocheae sp. nov. Sistema reproductor. Barra de escala $=1 \mathrm{~mm}$. Abreviaturas: am $=$ ampulla, $\mathrm{bc}=$ bursa copulatriz, $\mathrm{cd}=$ conducto deferente, $\mathrm{cv}=$ conducto vaginal, gf = glándula femenina, $\mathrm{pr}=$ próstata, $\mathrm{rs}=$ receptáculo seminal, $\mathrm{sp}=$ saco peneal, $\mathrm{v}=$ vagina. (Isid: zoobank. org: pub: 84145485-F43C-4C19-A0AB-5330C141B723)

Sistema nervioso.- (Figura 5) Presenta dos ganglios cerebrales y dos pleurales fusionados formando el complejo cerebro-pleural, sobre el cual se encuentran los ojos. Los ganglios pedales son más pequeños que el complejo cerebro-pleural y se encuentran unidos por comisuras viscerales. Tres nervios parten de los ganglios pedales y cuatro del complejo cerebro-pleural. Dos ganglios bucales se encuentran ventrales al esófago.

Etimología.- Trapania rocheae lleva el nombre de la Dra. Andrea Roche, mentora del primer autor de este trabajo. 


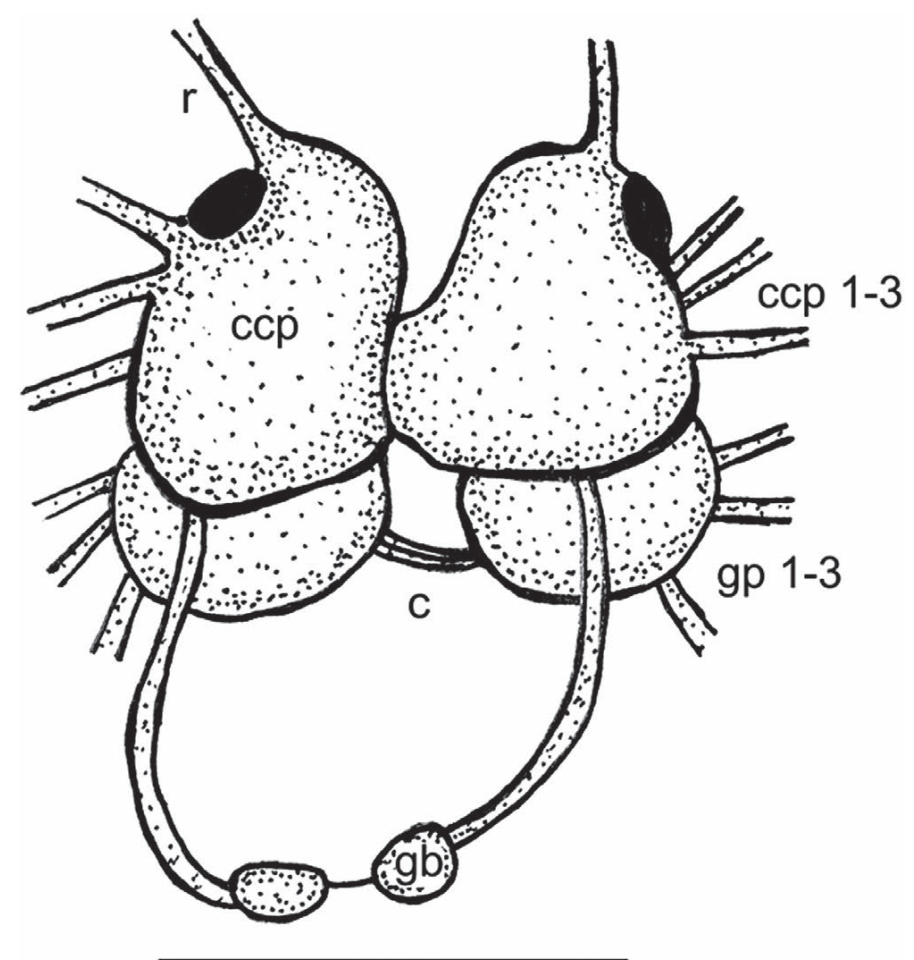

Figura 5. Trapania rocheae sp. nov. Sistema nervioso. Barra de escala $=0.5 \mathrm{~mm}$. Abreviaciones: $\mathrm{c}$

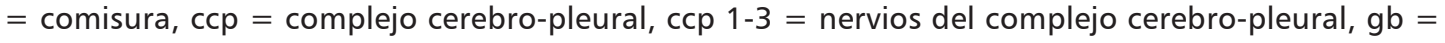
ganglio bucal, gp = ganglio pedal, gp 1-3 = nervios pedales, $r=$ nervio rinofórico. (Isid: zoobank. org: pub: 84145485-F43C-4C19-A0AB-5330C141B723).

\section{DISCUSIÓN}

Rudman (1987) señaló que hay dos conjuntos de caracteres útiles para distinguir las especies del género Trapania: la coloración externa y la morfología de los dientes radulares y mandíbulas. El género Trapania se encuentra representado por 46 especies (MolluscaBase, 2019) las cuales poseen patrones de coloración que no se asemejan al de Trapania rocheae sp. nov. Sobre la base de lo anterior T. rocheae sp. nov. se diferencia en ambos caracteres de las únicas dos especies sudamericanas $T$. darwini y $T$. maringa. Externamente T. darwini presenta una coloración blanquecina con parches de color marrón salpicados con manchas color crema además de la presencia de una coloración amarilla en las puntas de tentáculos orales, rinóforos, branquias, procesos extrarinofóricos y extrabranquiales y punta de la cola, las cuales no se encuentran presentes en T. rocheae sp. nov. Por otro lado, T. maringa no solo se diferencia de $T$. rocheae sp. nov. por la coloración predominante color marrón, sino también por las branquias que son bipinnadas y por la ausencia de la cresta notal. Internamente las rádulas de T. darwini y T. maringa presentan dentículos posteriores a la cúspide externa más desarrollada, los cuales no se observan en T. rocheae sp. nov.

Las especies descriptas para el Indo-Pacífico Oriental, África y Oceanía como: Trapania aureopunctata Rudman, 1987, T. toddi Rudman, 1987, T. aurata Rudman, 1987, T. davelli Rudman, 1987, T. rudmani Miller, 1981 y T. japonica Baba, 1935, tienen 
un cuerpo blanco manchado con amarillo y marrón. Otras, como T. brunnea Rudman, 1987, T. benni Rudman, 1987 y T. reticulata son marrones con manchas blancas y amarillas. Trapania luquei Ortea, 1989 predomina el negro en su cuerpo, mientras que T. pallida Kress, 1968 posee el cuerpo completamente blanquecino.

Incorporando la descripción de esta nueva especie, la familia Goniodorididae tiene dos especies en el Mar Argentino, Ancula fuegiensis Odhner, 1926 y Trapania rocheae sp. nov., la primera de ellas carece de registros en el Mar Argentino posteriores desde su descripción original por Odhner (1926) para Ushuaia, siendo registrada en el Pacífico Chileno por Fischer y Ortea (1996) y Schrördl (2003). Ambas especies presentan un patrón de coloración similar, difiriendo en la ubicación de las manchas que, en $A$. fuegiensis, se encuentran solo en el dorso alrededor de las branquias, mientras que en $T$. rocheae sp. nov. las manchas se extienden en todo el cuerpo incluido rinóforos, procesos digitiformes y branquias. Internamente las rádulas son diferentes, presentando $A$. fuegiensis un diente marginal, el cual está ausente en el género Trapania.

La combinación particular de caracteres que describimos en T. rocheae sp. nov. la distinguen como una nueva especie, representando una importante adición al conocimiento de los nudibranquios de esta región.

\section{AGRADECIMIENTOS}

Los autores agradecen la colaboración y el apoyo logístico de Cota Cero Buceo para la realización de este trabajo y al revisor anónimo por los comentarios realizados sobre la versión original del manuscrito.

\section{FINANCIAMIENTO}

Este estudio fue parcialmente soportado por PICT 2013-0197.

\section{PARTICIPACIÓN}

Los autores de este trabajo trabajamos en conjunto aportando de igual manera en el desarrollo de este estudio.

\section{LITERATURA CITADA / BIBLIOGRAPHY}

Ardila, N. E., Báez, D. P., Valdés, Á. (2007). Babosas y liebres de mar Mollusca: Gastropoda: Opisthobranchia) de Colombia. Biota Colombiana, 8 (2), 185-197.

Camacho-García, Y., Ortea, J. (2000). A new species of Trapania (Nudibranchia: Goniodorididae) from the Pacific coast of Central America. Revista de Biología Tropical 48, 317-322. 
Cervera, J. L., García-Gómez, J. C., Megina, C. (2000). A new species of Trapania Pruvot-Fol, 1931 from the Bay of Cadiz with remarks on other Trapania species. Ophelia, 52, 17-24.

Cervera, J. y García-Gomez, J.C. (1989). Dos nuevas especies de Trapania Pruvot-Fol, 1931 (Gastropoda: Nudibranchia) del sur de España. Bollettino Malacologico 24, 189-204.

Chavanich, S., Viyakarn, V., Sanpanich, K. Harris, L. G. (2013). Diversity and occurrence of nudibranchs in Thailand. Marine Biodiversity, 43 (1), 31-36.

Fahey, S. J. (2004). A new species of Trapania (Nudibranchia: Gonodorididae) from Western Australia with comparisons to other Indo-West Pacific Trapania. Zootaxa, 514 (1), 1-12.

Fischer, M. A., Ortea, J. (1996). New records of the genus Ancula Lovén, 1846 (Nudibranchia: Goniodorididae) on the American Pacific Coast. The Veliger, 39(1), 90-92.

García, F. J., Bertsch, H. (2009). Diversity and distribution of the Gastropoda Opisthobranchia from the Atlantic Ocean: a global biogeographic approach. Scientia Marina, 73 (1), 153-160.

Gosliner, T. M., Fahey, S. J. (2008). Systematics of Trapania (Mollusca: Nudibranchia: Goniodorididae) with descriptions of 16 new species. Systematics and Biodiversity, 6 (1), 53-98.

Haefelfinger, H. (1960). Neue und wenig bekannte Opisthobranchier der Gattungen Trapania und Caloria aus der Bucht von Villefranche-sur-Mer. Revue Suisse de Zoologie, 67, 226-238.

Hermosillo, A., Valdés, A. (2004). Two new species of Dorid Nudibranchs (Mollusca, Opisthobranchia) from Bahía de Banderas, Mexico. Proceedings of the California Academy of Sciences, 55, 550-560.

Holznagel, W. E. (1998). A nondestructive method for cleaning gastropod radulae from frozen, alcohol-fixed, or dried material. American Malacological Bulletin, $14,181-183$.

Kress, A. (1968). Trapania pallida sp. nov. (Opisthobranchia, Gastropoda), a genus new to Britain. Proceedings of the Malacological Society of London, 38, 161165.

Marcus, E. (1957). On opisthobranchia from Brazil (2). Zoological Journal of the Linnean Society, 43 (292), 390-486.

Millen, S., Bertsch, H. (2000). Three new species of Dorid Nudibranchs from Southern California, USA, and the Baja California peninsula, Mexico. The Veliger, 43, 354-366.

Miller, M. (1981). Trapania rudmani, a new Dorid Nudibranch (Gastropoda: Opisthobranchia) from New Zealand. New Zealand Journal of Zoology, 8, 5-9.

MolluscaBase. (2019). Trapania Pruvot-Fol, 1931. Accessed at: http://www.molluscabase.org/aphia.php? $\mathrm{p}=$ taxdetails\&id $=138043$ on $2019-10-02$

Odhner, N. (1926). Die Opisthobranchien. Further zoological results of the Swedish Antarctic expedition 1901-1903, 2 (1), 1-100.

Rudman, W.B. (1987). The genus Trapania (Nudibranchia: Goniodorididae) in the Indo-West Pacific. Journal of Molluscan Studies, 53, 189-212. 
Schrödl, M. (2003). Sea slugs of southern South America: systematics, biogeography and biology of Chilean and Magellanic Nudipleura (Mollusca-Opisthobranchia). ConchBooks, 173 pp. 